University of Nebraska - Lincoln

DigitalCommons@University of Nebraska - Lincoln

$5-1-2006$

\title{
Search for global-minimum geometries of medium-sized germanium clusters. II. Motif-based low-lying clusters $\mathrm{Ge}_{21}-\mathrm{Ge}_{29}$
}

S. Yoo

University of Nebraska-Lincoln

Xiao Cheng Zeng

University of Nebraska-Lincoln, xzeng1@unl.edu

Follow this and additional works at: https://digitalcommons.unl.edu/chemzeng

Part of the Chemistry Commons

Yoo, S. and Zeng, Xiao Cheng, "Search for global-minimum geometries of medium-sized germanium clusters. II. Motif-based low-lying clusters $\mathrm{Ge}_{21}-\mathrm{Ge}_{29}$ " (2006). Xiao Cheng Zeng Publications. 8.

https://digitalcommons.unl.edu/chemzeng/8

This Article is brought to you for free and open access by the Published Research - Department of Chemistry at DigitalCommons@University of Nebraska - Lincoln. It has been accepted for inclusion in Xiao Cheng Zeng Publications by an authorized administrator of DigitalCommons@University of Nebraska - Lincoln. 


\title{
Search for global-minimum geometries of medium-sized germanium clusters. II. Motif-based low-lying clusters $\mathrm{Ge}_{21}-\mathrm{Ge}_{29}$
}

\author{
S. Yoo and X. C. Zeng ${ }^{\text {a) }}$ \\ Department of Chemistry, University of Nebraska-Lincoln, Lincoln, Nebraska 68588
}

(Received 6 February 2006; accepted 13 March 2006; published online 9 May 2006)

\begin{abstract}
We performed a constrained search for the geometries of low-lying neutral germanium clusters $\mathrm{Ge}_{N}$ in the size range of $21 \leqslant N \leqslant 29$. The basin-hopping global optimization method is employed for the search. The potential-energy surface is computed based on the plane-wave pseudopotential density functional theory. A new series of low-lying clusters is found on the basis of several generic structural motifs identified previously for silicon clusters [S. Yoo and X. C. Zeng, J. Chem. Phys. 124, 054304 (2006)] as well as for smaller-sized germanium clusters [S. Bulusu et al., J. Chem. Phys. 122, 164305 (2005)]. Among the generic motifs examined, we found that two motifs stand out in producing most low-lying clusters, namely, the six/nine motif, a puckered-hexagonal-ring $\mathrm{Ge}_{6}$ unit attached to a tricapped trigonal prism $\mathrm{Ge}_{9}$, and the six/ten motif, a puckered-hexagonal-ring $\mathrm{Ge}_{6}$ unit attached to a bicapped antiprism $\mathrm{Ge}_{10}$. The low-lying clusters obtained are all prolate in shape and their energies are appreciably lower than the near-spherical low-energy clusters. This result is consistent with the ion-mobility measurement in that medium-sized germanium clusters detected are all prolate in shape until the size $N \sim 65$. () 2006 American Institute of Physics.
\end{abstract}

[DOI: $10.1063 / 1.2192783$ ]

\section{INTRODUCTION}

Among main group-IV elements, silicon and germanium have many properties in common. For example, both are semiconductors in cubic diamond form. Moreover, the global-minimum silicon clusters $\mathrm{Si}_{N}$ and germanium clusters $\mathrm{Ge}_{N}$ have identical geometries up to $N=12 .{ }^{1}$ In the size range of $13 \leqslant N \leqslant 20$, although the growth patterns of many silicon clusters differ from those of germanium clusters, ${ }^{1,2}$ a majority of low-lying silicon and germanium clusters still contain a common structural motif, namely, the tricapped trigonal prism (TTP) motif. ${ }^{3}$ In our previous study of germanium clusters in the size range of $16 \leqslant N \leqslant 20$ (Paper I of this series $^{2}$ ), we examined the second generic structural motif, namely, the so-called six/six motif ${ }^{4}$-a puckered-hexagonalring $\mathrm{Ge}_{6}$ unit attached to a tetragonal bipyramid $\mathrm{Ge}_{6}$. We found that some energetically competitive germanium clusters can be also built based on the six/six motif, such as $\mathrm{Ge}_{19}$. For silicon clusters, a TTP-to-six/six motif transition has been predicted to occur in the size range of $\mathrm{Si}_{16}$ to $\mathrm{Si}_{18}{ }^{4-7}$ However, for germanium clusters, the TTP motif dominates the population of the lowest-energy clusters in the size range of $10 \leqslant N \leqslant 20$, while the six/six motif plays a lesser role.

The silicon clusters in the size range of $21 \leqslant N \leqslant 29$ have received considerable attention over the past five years, ${ }^{4,8-17}$ largely because previous ion-mobility experiments ${ }^{18,19}$ showed that a structural transition from prolate to nearspherical geometries occurs at $N=27 \pm 2$ for both cation and anion silicon clusters. In contrast, for cation germanium clusters, previous ion-mobility experiments showed that the prolate-to-near-spherical transition does not occur until

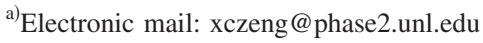

$N \sim 65 .^{20}$ Thus, it is expected that the growth pathway of silicon and germanium clusters would differ more from each other beyond the size range of $N=27-29$. Undoubtedly, if the geometries of lowest-energy germanium clusters in the size range of $21 \leqslant N \leqslant 29$ are known, it will help us to gain better insight into the structural evolution of small-sized clusters towards nanocrystalline quantum dots ${ }^{21}$ as well as to understand why the growth pathway of the two group-IV clusters starts to show major deviation beyond $N=29$. For the latter purpose, we first summarize some generic structural features of lowest- and low-lying silicon clusters in the size range of $21 \leqslant N \leqslant 30$. These features have been revealed by many researchers via either unbiased or constrained global searches in conjunction with either semiempirical tightbinding or the first-principles density functional theory (DFT) calculations of the potential-energy surface., ${ }^{910,12,13,17}$ Briefly, nearly all the low-lying silicon clusters in the size range of $21 \leqslant N \leqslant 30$ can be classified into four distinct structural families: ${ }^{17}$ (1) Clusters in the first family can be assembled by connecting two small-sized "magic-number" clusters $\mathrm{Si}_{N}(N=6,7,9$, or 10$)$ with a fused-puckeredhexagonal-ring $\mathrm{Si}_{9}$ unit (a fragment of bulk diamond silicon) in between; (2) clusters in the second family can be constructed on the basis of a structural motif consisting of a puckered-hexagonal-ring $\mathrm{Si}_{6}$ and small-sized magic-number clusters $\operatorname{Si}_{N}(N=6,7,9$, and 10), that is, the six/six, six/seven, six/nine, or six/ten motif; (3) clusters in the third family are near spherical cagelike in geometry, and many can be viewed as homologue to the endohedral carbon fullerenes; ${ }^{12}$ and (4) clusters in the fourth family are Y-shaped "three-arm" clusters, in which the three arms are the small-sized magic clusters and the "glue" unit is structurally similar to the fusedpuckered-hexagonal-ring $\mathrm{Si}_{9}$ unit. 
The purpose of this paper is to examine relative stability of those low-lying germanium clusters with structures homologues to the four silicon structural families discussed above. We noticed that compared to the silicon clusters, much less theoretical studies have been devoted to the structures of low-lying germanium clusters. ${ }^{1,22-24}$ To our knowledge, only two papers in the literature show geometric structures of low-lying $\mathrm{Ge}_{N}$ in the size range of $21 \leqslant N \leqslant 29$. Wang et al. reported candidates for lowest-energy clusters up to $N=25$, based on a global search using the genetic algorithm (GA) coupled with a nonorthogonal tight-binding (NTB) model. ${ }^{23}$ They found that for $\mathrm{Ge}_{N}(N>13)$, the stacked layered structures and the spherical-like compact structures compete for the lowest-energy structures. Later, Liang and $\mathrm{Li}$ studied geometric structures and electronic properties of mediumsized clusters $\mathrm{Ge}_{N}(21 \leqslant N \leqslant 25)$ using a full-potential linearmuffin-tin-orbital molecular-dynamics (FP-LMTO-MD) method. ${ }^{24}$ They suggested that low-lying prolate clusters can be built upon stacked TTP motifs. In this work, we combined the basin-hopping global optimization method with DFT calculation of the potential-energy surface. This combined computational approach coupled with many structural motifs identified previously allows us to obtain many new low-lying clusters with energy appreciably lower than the corresponding isomers reported previously. ${ }^{22-25}$

\section{METHOD}

We used the basin-hopping global optimization method to search for the geometries of low-lying clusters. The basinhopping $(\mathrm{BH})$ method has been applied to the Lennard-Jones clusters $^{26-28}$ as well as other atomic and molecular clusters. ${ }^{27,29-32}$ Here we briefly summarize the BH global optimization technique. Details of this technique can be found elsewhere. ${ }^{26,27,33}$ A key idea of the $\mathrm{BH}$ method is that the transformed potential-energy surface (PES) $\widetilde{U}$ can be generated via the mapping

$$
\tilde{U}\left(N, \mathbf{r}_{1}, \mathbf{r}_{2}, \ldots, \mathbf{r}_{N}\right)=\min \left\{U\left(N, \mathbf{r}_{1}, \mathbf{r}_{2}, \ldots, \mathbf{r}_{N}\right)\right\},
$$

where min denotes the energy minimization performed with starting configuration of $\left\{\mathbf{r}_{1}, \cdots, \mathbf{r}_{N}\right\}$ and $U$ is the PES. The topography of the transformed PES resembles a multidimensional staircase where each step corresponds to the basin of attraction. The basin of attraction represents a set of geometries from which the energy minimization always leads to the local minimum. By removing the intra-potential-well vibration, the system can "hop" directly between local minima at each step.

In practice, $\widetilde{U}$ can be explored via canonical Monte Carlo (MC) simulation. For example, at each MC trial all movable coordinates are randomly displaced with an adjustable step size to yield an acceptance ratio of 0.5. The energy change $\Delta \widetilde{U}$ for hopping between two minima is accepted with the probability of $\exp \left(-\Delta \widetilde{U} / k_{B} T\right)$. In our previous study of smaller germanium clusters ${ }^{2}$ (Paper I), we found that by directly coupling the $\mathrm{BH}$ method with the density functional calculation of the PES, we can generate low-lying clusters with energy appreciably lower than those reported previously

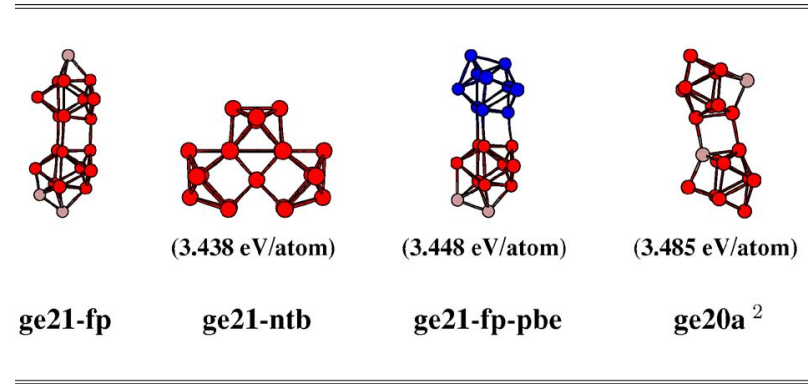

FIG. 1. (Color online) Geometries of two lowest-energy $\mathrm{Ge}_{21}$ clusters previously reported: ge21-fp (Ref. 24) and ge21-ntp (Ref. 23). The cluster ge21-fp-pbe is obtained via a geometric optimization based on DFT/PBE calculation with the ge21-fp as the initial structure. The binding energies per atom (shown in parentheses) are based on the DFT/PBE calculation. The TTP $\mathrm{Ge}_{9}$ motif and the bicapped antiprism $\mathrm{Ge}_{10}$ motif are highlighted in red and blue, respectively. To compare the binding energy per atom, the lowestenergy cluster $\mathrm{Ge}_{20}$ (Ref. 2) is also displayed.

based on semiempirical tight-binding model. ${ }^{23}$ Here, we used the plane-wave-basis pseudopotential method with two popular exchange-correlation functionals within the general gradient approximation (GGA) - the Perdew-BurkeErnzerhof (PBE) functional ${ }^{34}$ and the BLYP functional ${ }^{35}$-implemented in the CPMD code. ${ }^{36}$ We chose the norm-conserving nonlocal pseudopotential ${ }^{37}$ constructed in the scheme of Troullier and Martins as a separable form of that of Kleinman-Bylander. ${ }^{38,39}$ Initially, the constrained global search was done with less accurate PES for which a relatively small super-cell-size (20 $\AA$ ) and plane-wave cutoff (15 Ry) were used. Once the top-five low-lying isomers were generated, their geometric structures were reoptimized by using a larger super-cell-size $(25 \AA)$ and plane-wave cutoff (30 Ry). No symmetry constraint was enforced during the geometry optimization.

Since DFT calculation of the PES demands much more computational time than the tight-binding calculations, a full (unbiased) global search for germanium clusters with size $N \geqslant 20$ becomes nearly impractical with our current computer facility. As shown previously, ${ }^{4}$ if some generic structural motifs can be identified from smaller low-lying clusters, motif-based (constrained) global search can greatly reduce the computational time. In practice, the structure of the motif can be fixed while only remaining atoms of the clusters are subject to the MC trial move. However, we can no longer claim the lowest-energy clusters obtained from the constrained search as the global minima.

\section{RESULTS AND DISCUSSION}

First, we have examined binding energies per atom of two previously reported lowest-energy $\mathrm{Ge}_{21}$ clusters, one obtained based on the combined genetic algorithm/NTB $\operatorname{search}^{23}$ and another obtained via FP-LMTO-MD simulation. ${ }^{24}$ Both global searches suggested that the lowestenergy $\mathrm{Ge}_{21}$ cluster should contain the TTP motif. The two candidates for the global minimum of $\mathrm{Ge}_{21}$ are shown in Fig. 1 where the cluster based on the FP-LMTO-MD calculation is denoted as ge21-fp and the one based on the NTB calculation is denoted as ge21-ntb. Both clusters contain the TTP motifs (highlighted in red). We reoptimized geometric struc- 
TABLE I. Calculated energy differences with respect to the corresponding lowest-energy clusters (denoted by the boldfaced value $\mathbf{0 . 0 0 0}$ ). Binding energy per atom (in eV/atom) of the lowest-energy clusters is given in parentheses. Those isomers whose energy-difference value from the lowestenergy isomer is less than $0.1 \mathrm{eV}$ are considered to be isoenergetic with the lowest-energy cluster and their energies are also highlighted in boldface.

\begin{tabular}{|c|c|c|}
\hline & PBE $(e V)$ & BLYP (eV) \\
\hline ge21-1a & 0.098 & $\mathbf{0 . 0 0 0}(3.177)$ \\
\hline ge21-2a & $\mathbf{0 . 0 0 0}(3.476)$ & 0.167 \\
\hline ge $21-2 b$ & 0.160 & 0.185 \\
\hline ge21-ntb & 0.793 & 1.041 \\
\hline ge22-1a & 0.481 & 0.131 \\
\hline ge22-2a & $\mathbf{0 . 0 0 0}(3.487)$ & $\mathbf{0 . 0 0 0}(3.178)$ \\
\hline ge $22-2 b$ & 0.022 & 0.029 \\
\hline ge22-ntb & 1.816 & 2.113 \\
\hline ge23-1a & 0.887 & 0.531 \\
\hline ge23-2a & $\mathbf{0 . 0 0 0}(3.495)$ & 0.010 \\
\hline ge $23-2 b$ & 0.005 & $\mathbf{0 . 0 0 0}(3.188)$ \\
\hline ge $23-2 c$ & 0.111 & 0.029 \\
\hline ge23-ntb & 1.438 & 1.665 \\
\hline ge24-1a & 0.358 & 0.367 \\
\hline ge24-2a & $\mathbf{0 . 0 0 0}(3.449)$ & $\mathbf{0 . 0 0 0}(3.197)$ \\
\hline ge $24-2 b$ & 0.210 & 0.355 \\
\hline ge24-ntb & 1.463 & 1.807 \\
\hline ge25-1a & 0.001 & $\mathbf{0 . 0 0 0}(3.198)$ \\
\hline ge25-2a & 0.000 & 0.144 \\
\hline ge $25-2 b$ & 0.070 & 0.194 \\
\hline ge25-ntb & 1.812 & 2.392 \\
\hline ge26-1a & 0.390 & 0.171 \\
\hline ge26-2a & $0.000(3.511)$ & $\mathbf{0 . 0 0 0}(3.200)$ \\
\hline ge26-3a & 1.079 & 1.712 \\
\hline ge26-4a & 0.969 & 0.640 \\
\hline ge27-1a & $\mathbf{0 . 0 0 0}(3.494)$ & $\mathbf{0 . 0 0 0}(3.184)$ \\
\hline ge27-1b & 0.366 & 0.206 \\
\hline ge27-3a & 0.779 & 1.415 \\
\hline ge27-4a & 0.647 & 0.297 \\
\hline ge28-1a & $\mathbf{0 . 0 0 0}(3.509)$ & $\mathbf{0 . 0 0 0}(3.199)$ \\
\hline ge $28-3 a$ & 1.324 & 2.0016 \\
\hline ge28-4a & 1.381 & 1.066 \\
\hline ge29-1a & $\mathbf{0 . 0 0 0}(3.524)$ & $\mathbf{0 . 0 0 0}(3.212)$ \\
\hline ge29-3a & 1.725 & 2.575 \\
\hline ge29-4a & 1.032 & 0.848 \\
\hline
\end{tabular}

tures of both ge21-fp and ge21-ntb using the PBE functional. The geometry of ge21-fp undergoes a notable change-the capped TTP subunit is converted into $\mathrm{Ge}_{10}$ bicapped antiprism $^{40}$ as shown in blue for ge21-fp-pbe (Fig. 1). Our DFT/PBE calculation indicates that both low-lying clusters have binding energy per atom about $0.037-0.047 \mathrm{eV} /$ atom less than the proposed global-minimum cluster ge20a reported in Ref. 2, indicating that the two low-lying isomers are not the global minimum. Furthermore, as shown in Table I (see below), a newly obtained lowest-energy cluster (ge21-2a) is $0.793 \mathrm{eV}$ lower in energy than ge21-ntb.

In Fig. 2 we display representative clusters examined in this study. As discussed in the Introduction, our main focus is placed on four distinct structural families (which have been previously studied for the silicon counterparts ${ }^{17}$ ): (1) Prolate clusters in family I can be assembled by connecting two small-sized magic-number clusters-either the tetragonal bipyramid $\mathrm{Ge}_{6}$ (yellow), pentagonal bipyramid $\mathrm{Ge}_{7}$ (light-purple), TTP $\mathrm{Ge}_{9}$ (red), or bicapped antiprism $\mathrm{Ge}_{10}$ (blue)-via a fused-puckered-hexagonal-ring $\mathrm{Ge}_{9}$ unit (green); (2) prolate clusters in family II can be constructed on the basis of a structural motif consisting of a puckeredhexagonal-ring $\mathrm{Ge}_{6}$ (green) and small-sized magic-number clusters $\mathrm{Ge}_{N}(N=6,7,9$, and 10), that is, either the six/six, six/seven, six/nine, or six/ten motif; (3) near-spherical clusters in family III are endohedral cagelike clusters (endohedral atoms are in blue); ${ }^{12}$ and (4) clusters in family IV are "handmade" Y-shaped three-arm clusters, in which the three arms are the small-sized clusters $\left(\mathrm{Ge}_{6}-\mathrm{Ge}_{9}\right)$ and the glue unit (in green) is structurally similar to the fused-puckeredhexagonal-ring $\mathrm{Ge}_{9}$ unit. In Fig. 2, we also used the same cluster notation as before, ${ }^{17}$ where the notation 1a refers to the isomer that has the lowest (PBE) energy in family I and the notation $1 \mathrm{~b}$ refers to the isomer that has the second lowest energy in family I. In Table I, we list the binding energies per atom of the lowest-energy clusters and the energy difference between all clusters with respect to the lowest-energy clusters. It can be seen that the binding energies per atom increase rather smoothly with the increase of cluster size $N$. For comparison, we also displayed those lowest-energy clusters $\left(\mathrm{Ge}_{21}-\mathrm{Ge}_{25}\right)$ reported by Wang et al. ${ }^{23}$ with the cluster notation ge21-ntb to ge25-ntb. As shown in Table I, the NTB global minima are typically $1-2 \mathrm{eV}$ higher in energy than the corresponding lowest-energy isomers in family I or II, indicating that improved NTB parameters may be needed to accurately compute the PES of germanium clusters.

Some major structural differences between the low-lying germanium clusters and silicon counterparts in the size range of $21 \leqslant N \leqslant 29$ can be summarized as follows.

(1) The endohedral cagelike near-spherical clusters of family III are not energetically competitive compared to the prolate clusters in family I or II. Typically, nearspherical clusters are $0.7-2 \mathrm{eV}$ higher in energy than the prolate counterparts, regardless of the DFT/PBE or DFT/BLYP calculations. In contrast, for neutral silicon clusters, near-spherical clusters become competitive in energy compared with the prolate isomers, starting from $\mathrm{Si}_{26}$. To obtain low-energy near-spherical germanium clusters, we first set the initial structures of nearspherical germanium clusters to be the same as those of the lowest-energy silicon clusters. ${ }^{17}$ We then performed BH/DFT-PBE search using $50 \mathrm{MC}$ trials to obtain slightly improved local minima of near-spherical clusters. Still, we found that the prolated isomers are notably lower in energy than all the near-spherical isomers obtained. This result is consistent with the ion-mobility measurements in that the prolate-to-near-spherical structural transformation does not occur for germanium clusters until $N \sim 65{ }^{20}$

(2) The Y-shaped three-arm clusters in family IV are not energetically competitive compared to the prolate clusters in family I or II. Only ge27-4a can be viewed as a low-lying isomer since DFT/BLYP calculation shows that it is only about $0.3 \mathrm{eV}$ higher in energy than the lowest-energy isomer. 


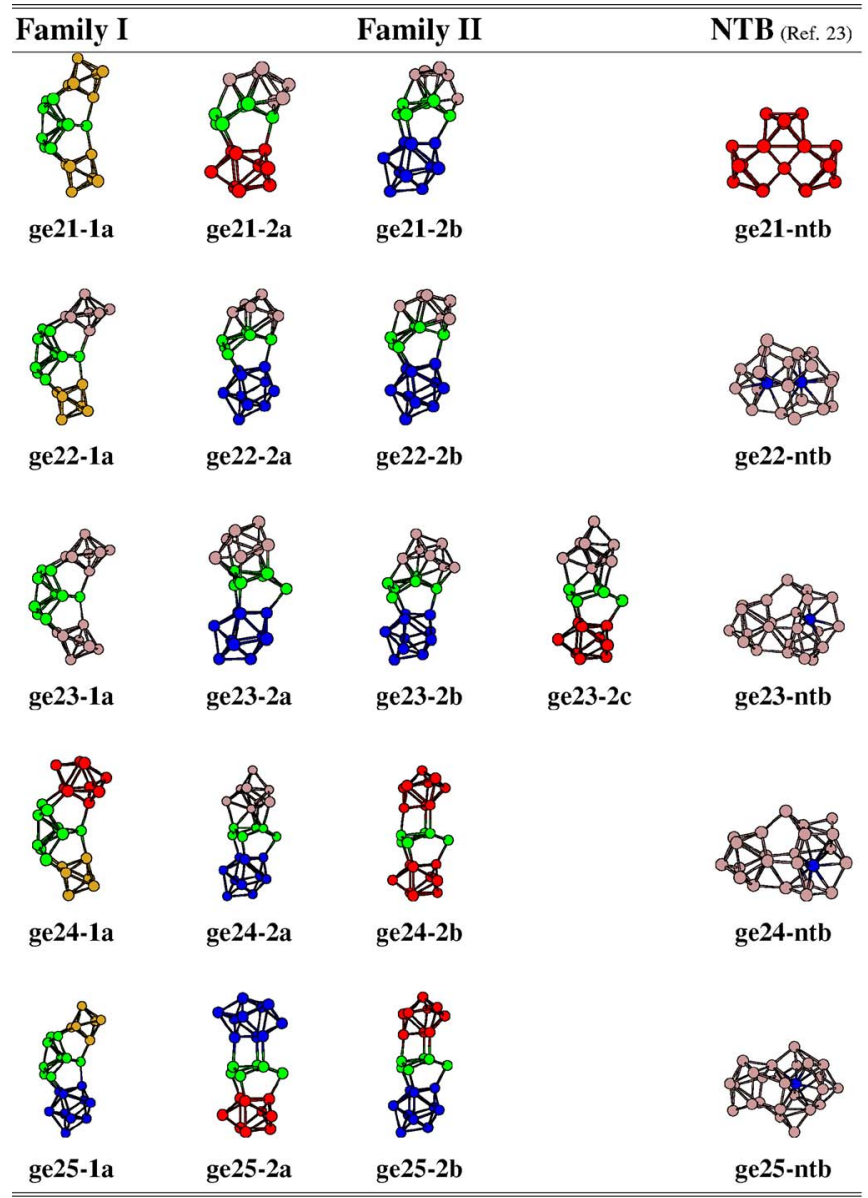

(a)
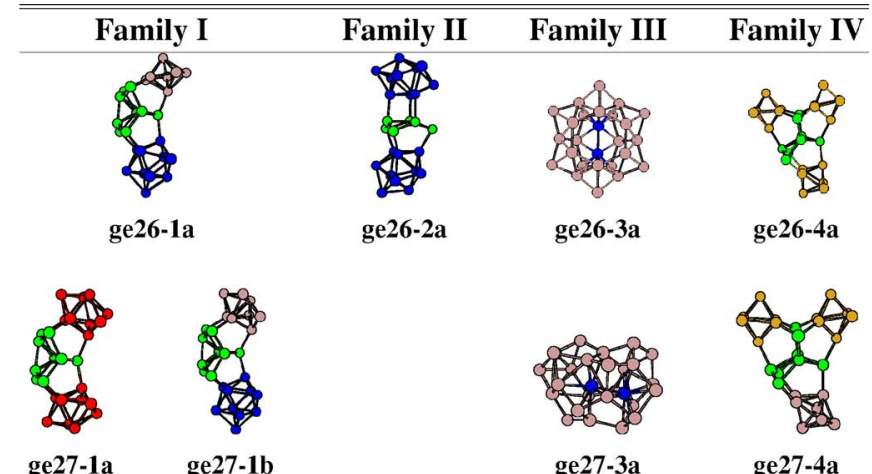

ge27-3a

ge27-4a
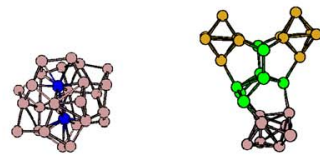

ge28-3a

ge28-4a

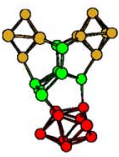

ge29-4a

FIG. 2. (Color online) Low-energy clusters of (a) $\mathrm{Ge}_{N}(21 \leqslant N \leqslant 25)$ and (b) $\mathrm{Ge}_{N}(26 \leqslant N \leqslant 29)$ based on the constrained basin-hopping global search. The prolate clusters in families I and II as well as the "three-arm" clusters in family IV are built upon various generic structural motifs (Ref. 17). The puckered-hexagonal-ring $\mathrm{Ge}_{6}$ unit and the fused-puckered-hexagonal-ring $\mathrm{Ge}_{9}$ unit are highlighted in green. The tetragonal bipyramid $\mathrm{Ge}_{6}$, pentagonal bipyramid $\mathrm{Ge}_{7}$, TTP $\mathrm{Ge}_{9}$, and bicapped antiprism $\mathrm{Ge}_{10}$ are highlighted in yellow, light-purple, red, and blue, respectively. For endohedral cagelike clusters, the endohedral atoms are highlighted in blue. The notation 1a refers to the isomer that has the lowest energy (DFT/PBE calculation) in family I and the notation $\mathbf{1 b}$ refers to the isomer that has the second lowest-energy in family I. The lowest-energy germanium clusters obtained based on the NTB calculations (Ref. 23) are also displayed in (a).

(3) Among the prolate clusters in families I and II, DFT/PBE calculation favors the family II clusters $\left(\mathrm{Ge}_{21}-\mathrm{Ge}_{26}\right)$ which all contain either a TTP motif or a $\mathrm{Ge}_{10}$ motif. For $\mathrm{Ge}_{21}$ and $\mathrm{Ge}_{25}$ DFT/BLYP calculation suggests that clusters ge21-1a and ge25-1a in family I are the lowest-energy clusters. These results are consistent with those of silicon clusters ${ }^{7,17}$ in that DFT/BLYP total-energy calculation slightly favors the six/six motif whereas DFT/PBE calculation favors the TTP motif. Note that because of the intrinsic error bar of DFT total-energy calculation (typically within $5 \mathrm{meV} / \mathrm{atom}$ in binding energy per atom for semiconductor clusters), we can view that those isomers with the energy difference from the lowest-energy isomer less than $0.1 \mathrm{eV}$ (see Table I) are isoenergetic with the lowest-energy isomer. In other words, these "isoenergetic" isomers can be all viewed as leading candidates for the global minimum. For example, ge22-2a and ge22-2b are isoenergetic, and so are ge23-2a, ge23-2b, and ge23-2c. Their energy differences are highlighted in bold in Table I.
(4) The last main structural feature of the lowest- and lowlying germanium clusters is that several clusters with an odd number of atoms (ge21-2a, ge25-2a, and ge271a) contain the six/nine $\left(\mathrm{Ge}_{6} / \mathrm{Ge}_{9}\right)$ motif in which the nine-atom subunit is the TTP unit. As a comparison, many silicon-cluster counterparts favor the six/ten $\left(\mathrm{Ge}_{6} / \mathrm{Ge}_{10}\right)$ motif (except $\left.\mathrm{Si}_{23}{ }^{17}\right)$. Similar conclusion has been made before for smaller germanium clusters, ${ }^{2}$ that is, the TTP motif is strongly favored by the lowlying germanium clusters compared to the silicon clusters. For germanium clusters with an even number of atoms, the six/ten $\left(\mathrm{Ge}_{6} / \mathrm{Ge}_{10}\right)$ structural motif is universally favored, as for the silicon clusters.

\section{CONCLUSION}

We have performed a constrained global search for the geometries of low-lying germanium clusters in the size range of $21 \leqslant N \leqslant 29$. Because of the constrained search in nature, we cannot claim that the lowest-energy clusters obtained are the global minima. At present, we can only consider these 
lowest-energy clusters as leading candidates for the global minima since they are all appreciably lower in energy than those previously reported based on either NTB calculations or DFT calculations. Several distinct structural features of the germanium clusters are identified and their main differences from the silicon cluster in the same size range are discussed. First and foremost, the endohedral cagelike nearspherical germanium clusters are not energetically competitive in this size range. In other words, the low-lying germanium clusters in the size range of $21 \leqslant N \leqslant 29$ are all prolate in shape. This result is consistent with the ion-mobility measurement in that medium-sized clusters detected are prolate in shape for $N<65$. Second, the six/nine motif-a puckeredhexagonal-ring $\mathrm{Ge}_{6}$ unit attached to a tricapped trigonal prism $\mathrm{Ge}_{9}$ - is strongly favored by low-lying germanium clusters with an odd number of atoms, whereas the six/ten motif is universally favored by the germanium clusters with an even number of atoms. In contrast, many low-lying silicon clusters favor the six/six motif, rather than the six/nine motif. One way to confirm the existence of these generic structural motifs is to compare simulated anion photoelectron spectra and photoionization thresholds with the measured ones, ${ }^{41}$ as recently done for silicon clusters. ${ }^{16}$ Such a joint experimental/theoretical effort is underway.

\section{ACKNOWLEDGMENTS}

We thank Professor L. S. Wang, Dr. J. L. Wang, and Dr. J. J. Zhao for valuable discussions. This research was supported by grants from DOE (DE-FG02-04ER46164) and the Nebraska Research Initiative, by the John Simon Guggenheim Memorial Foundation, and by the Research Computing Facility at University of Nebraska-Lincoln.

${ }^{1}$ A. A. Shvartsburg, B. Liu, Z.-Y. Lu, C.-Z. Wang, M. F. Jarrold, and K.-M. Ho, Phys. Rev. Lett. 83, 2167 (1999).

${ }^{2}$ S. Bulusu, S. Yoo, and X. C. Zeng, J. Chem. Phys. 122, 164305 (2005).

${ }^{3}$ K.-M. Ho, A. A. Shvartsburg, B. Pan, Z.-Y. Lu, C.-Z. Wang, J. G. Wacker, J. L. Fye, and M. F. Jarrold, Nature (London) 392, 582 (1998).

${ }^{4}$ S. Yoo and X. C. Zeng, Angew. Chem., Int. Ed. 44, 1491 (2005).

${ }^{5}$ I. Rata, A. A. Shvartsburg, M. Horoi, Th. Frauenheim, K. W. Michael Siu, and K. A. Jackson, Phys. Rev. Lett. 85, 546 (2000).

${ }^{6}$ S. Goedecker, W. Hellmann, and T. Lenosky, Phys. Rev. Lett. 95, 055501 (2005).

${ }^{7}$ S. Yoo and X. C. Zeng, J. Chem. Phys. 123, 164303 (2005).
${ }^{8}$ L. Mitas, J. C. Grossman, I. Stich, and J. Tobik, Phys. Rev. Lett. 84, 1479 (2000)

${ }^{9}$ B.-X. Li, P.-L. Cao, and S.-C. Zhan, Phys. Lett. A 316, 252 (2003); B.-X. Li, Phys. Rev. B 71, 235311 (2005).

${ }^{10}$ A. Sieck, Th. Frauenheim, and K. A. Jackson, Phys. Status Solidi B 240, 537 (2003).

${ }^{11}$ S. Yoo, X. C. Zeng, X. Zhu, and J. Bai, J. Am. Chem. Soc. 125, 13318 (2003).

${ }^{12}$ S. Yoo, J. J. Zhao, J. L. Wang, and X. C. Zeng, J. Am. Chem. Soc. 126, 13845 (2004).

${ }^{13}$ K. A. Jackson, M. Horoi, I. Chaudhuri, Th. Frauenheim, and A. A. Shvartsburg, Phys. Rev. Lett. 93, 013401 (2004).

${ }^{14}$ A. A. Shvartsburg, M. Horoi, and K. A. Jackson, Spectroscopy of Emerging Materials (Kluwer, Dordrecht, 2004).

${ }^{15}$ O. Guliamov, L. Kronik, and K. A. Jackson, J. Chem. Phys. 123, 204312 (2005).

${ }^{16}$ J. Bai, L.-F. Cui, J. L. Wang, S. Yoo, X. Li, J. Jellinek, C. Koehler, T. Frauenheim, L.-S. Wang, and X. C. Zeng, J. Phys. Chem. A 110, 908 (2006).

${ }^{17}$ S. Yoo and X. C. Zeng, J. Chem. Phys. 124, 054304 (2006).

${ }^{18}$ M. F. Jarrold and V. A. Constant, Phys. Rev. Lett. 67, 2994 (1991).

${ }^{19}$ A. A. Shvartsburg, R. R. Hudgins, P. Dugourd, and M. F. Jarrold, Chem. Soc. Rev. 30, 26 (2001).

${ }^{20}$ J. M. Hunter, J. L. Fye, M. F. Jarrold, and J. E. Bower, Phys. Rev. Lett. 73, 2063 (1994).

${ }^{21}$ A. P. Alivisatos, Science 271, 933 (1996).

${ }^{22}$ C. Jo and K. Lee, J. Chem. Phys. 113, 7268 (2000).

${ }^{23}$ J. Wang, G. Wang, and J. Zhao, Phys. Rev. B 64, 205411 (2001).

${ }^{24}$ F.-S. Liang and B.-X. Li, Phys. Lett. A 328, 407 (2004).

${ }^{25}$ J. Wang, M. Yang, G. H. Wang, and J. J. Zhao, Chem. Phys. Lett. 367, 448 (2003).

${ }^{26}$ D. J. Wales and J. P. K. Doye, J. Phys. Chem. A 101, 5111 (1997).

${ }^{27}$ J. P. K. Doye and D. J. Wales, Phys. Rev. Lett. 80, 1357 (1998).

${ }^{28}$ J. P. K. Doye, M. A. Miller, and D. J. Wales, J. Chem. Phys. 110, 6896 (1999).

${ }^{29}$ D. J. Wales, Science 271, 925 (1996).

${ }^{30}$ D. J. Wales and M. P. Hodges, Chem. Phys. Lett. 286, 65 (1998).

${ }^{31}$ D. J. Wales, M. A. Miller, and T. R. Walsh, Nature (London) 394, 758 (1998).

${ }^{32}$ D. J. Wales and H. A. Scheraga, Science 285, 1368 (1999).

${ }^{33}$ D. J. Wales, Energy Landscapes with Applications to Clusters, Biomolecules and Glasses (Kluwer, Berlin, 2004).

${ }^{34}$ J. Perdew, K. Burke, and M. Ernzerhof, Phys. Rev. Lett. 77, 3865 (1996).

${ }^{35}$ A. D. Becke, Phys. Rev. A 38, 3098 (1988); C. Lee, W. Yang, and R. G. Parr, Phys. Rev. B 37, 785 (1988).

${ }^{36}$ J. Hutter, A. Alavi, T. Deutsch, M. Bernasconi, S. Goedecker, D. Marx, M. Tuckerman, and M. Parrinello, CPMD, Version 3.7.1, MPI für Festkörperforschung, Stuttgart, 1997-2001.

${ }^{37}$ D. Hamann, Phys. Rev. B 40, 2980 (1989).

${ }^{38}$ N. Troullier and J. L. Martins, Phys. Rev. B 43, 1993 (1991).

${ }^{39}$ L. Kleinman and D. M. Bylander, Phys. Rev. Lett. 48, 1425 (1982).

${ }^{40}$ S. Ogöt and J. R. Chelikowsky, Phys. Rev. B 55, R4914 (1997).

${ }^{41}$ S. Yoshida and K. Fuke, J. Chem. Phys. 111, 3880 (1999). 